\title{
ON JORIS' THEOREM ON DIFFERENTIABILITY OF FUNCTIONS
}

\author{
By ICHIRo Amemiya AND KazUo MASUdA
}

\section{Introduction.}

Let $f: \boldsymbol{R}^{n} \rightarrow \boldsymbol{R}$ be a function. If $f^{2}, f^{3} \in C^{\infty}$, does it follow that $f \in C^{\infty}$ ? The Inverse Function Theorem does not immediately give the answer. In 1982 $\mathrm{H}$. Joris answered this problem affirmatively by showing the following theorem.

TheOREM 1 (H. Joris [J]). Let $n_{1}, n_{2}, \cdots, n_{m}$ be positive integers with g.c.d. $\left\{n_{1}, n_{2}, \cdots, n_{m}\right\}=1$. If $f: \boldsymbol{R}^{n} \rightarrow \boldsymbol{R}$ is a function such that $f^{n_{2}} \in C^{\infty}$ for $i=1,2, \cdots, m$, then $f \in C^{\infty}$.

In the same paper $\mathrm{H}$. Joris proposed the next problem.

Problem. Find the other families of smooth functions $\left\{\boldsymbol{\phi}_{i}: \boldsymbol{R} \rightarrow \boldsymbol{R} \mid i=1,2, \cdots, m\right\}$ having the following property: For any function $f: \boldsymbol{R}^{n} \rightarrow \boldsymbol{R}, f$ is smooth if and only if $\phi_{i} \circ f$ is smooth for $i=1,2, \cdots, m$.

If we assume the continuity of $f$, then we need only consider the germs at $x=0$ since the study of differentiability is a local problem. In $1985 \mathrm{~J}$. Duncan, S. G. Krantz and H.R. Parks gave a certain family $\left\{\phi_{i}\right\}$ for continuous $f$.

Theorem 2 (J. Duncan, S. G. Krantz and H. R. Parks, [D] Theorem 2). Let $\phi_{i}: \boldsymbol{R} \rightarrow \boldsymbol{R}$ be smooth functions such that $\phi_{i}(x)=x^{n_{2}}+$ "higher order terms" near $x=0$ for $i=1,2, \cdots, m$ with g.c.d. $\left\{n_{1}, n_{2}, \cdots, n_{m}\right\}=1$. Then $\left\{\phi_{i}\right\}$ has the following property: For any continuous function $f: \boldsymbol{R}^{n} \rightarrow \boldsymbol{R}$ with $f(0)=0, f$ is smooth near $x=0$ if and only if $\phi_{i}^{\circ} f$ is smooth near $x=0$ for $i=1,2, \cdots, m$.

In the present paper, we give a simple proof of Joris' Theorem $(\S 2)$ and the necessary and sufficient condition for $\left\{\phi_{i}\right\}$ to have the property mentioned in Theorem 2 ( $\$ 3$ Theorem 3 ). In Appendix ( $\$ 4)$, we discuss this condition further, especially for polynomials $\phi_{i}$.

\section{Simple proof of Joris' Theorem.}

The essential part of our proof is the following algebraic lemma.

Received August 4, 1988 
LEMMA 1. Let $\mathscr{R}$ be a ring and $\mathcal{S}$ a subring of $\mathcal{R}$ with the property:

$(P)$ if $a \in \mathscr{R}$ and $a^{r} \in \mathcal{S}$ for every sufficiently large positive integer $r$, then $a \in \mathcal{S}$. Then the ring $\mathcal{S} \llbracket x \rrbracket$ of formal power series of $x$ with coefficients in $\mathcal{S}$ has the property $(P)$ as a subring of $\mathcal{R} \llbracket x \rrbracket$.

Proof. Suppose $f=\sum_{\imath=0}^{\infty} a_{\imath} x^{k+\imath}$ for some $k \geqq 0, a_{i} \in \mathcal{R}$, and $f^{r} \in \mathcal{S} \llbracket x \rrbracket$ for every large $r$, then since $a_{0}^{r}$ is the coefficient of the lowest term of $f^{r}, a_{0}^{r} \in \mathcal{S}$ and hence by $(P) a_{0} \in \mathcal{S}$. The set $\mathcal{S}^{\prime}$ of all the elements $a$ of $\mathscr{R}$ for which $a_{0} a^{m}$ $\in \mathcal{S}$ for every positive integer $m$ is a subring of $\mathcal{R}$ including $\mathcal{S}$, because $a_{0} a^{r m}$, $a_{0} b^{r m} \in \mathcal{S}$ implies $a_{0}^{r} a^{r m} b^{r m} \in \mathcal{S}$ for $r \geqq 2$ and hence $a_{0}(a b)^{m} \in \mathcal{S}$. Now we prove, by induction, $a_{n} \in \mathcal{S}^{\prime}$ for every $n$. Suppose $a_{0}, a_{1}, \cdots, a_{n} \in \mathcal{S}^{\prime}$, then for $f_{n}=\sum_{\imath=0}^{n} a_{\imath} x^{k+\imath}$, $a_{0} f_{n}^{m} \in \mathcal{S} \llbracket x \rrbracket$ for every $m$. So $a_{0} f^{r}\left(f-f_{n}\right)^{(r+1) m}=\sum_{s}$ const. $f^{r+s} a_{0} f_{n}^{(r+1) m-s} \in \mathcal{S} \llbracket x \rrbracket$ and hence the coefficient $a_{0}^{r+1} a_{n+1}^{(r+1) m}$ of $x^{k r+(k+n+1)(r+1) m}$ in $a_{0} f^{r}\left(f-f_{n}\right)^{(r+1) m}$ is in $\mathcal{S}$. Therefore, by $(P), a_{0} a_{n+1}^{m} \in \mathcal{S}$, that is, $a_{n+1} \in \mathcal{S}^{\prime}$. Since $a_{n} \in \mathcal{S}^{\prime}$ for every $n$, $a_{0} f^{m} \in \mathcal{S} \llbracket x \rrbracket$ for every $m$. Then for $f-a_{0} x^{k}=a_{1} x^{k+1}+\cdots,\left(f-a_{0} x^{k}\right)^{r}=f^{r}+$ $\sum_{s=1}^{r}$ const. $a_{0}^{s} f^{r-s} x^{k s} \in \mathcal{S} \llbracket x \rrbracket$. This shows $a_{1} \in \mathcal{S}$ and repeating the same argument, we have $a_{n} \in \mathcal{S}$ for every $n$, that is, $f \in \mathcal{S} \llbracket x \rrbracket$, which completes the proof of Lemma 1.

Let $f: \boldsymbol{R}^{n} \rightarrow \boldsymbol{R}$ be a function such that $f^{n_{\imath} \in C^{\infty}}$ for $i=1,2, \cdots, m$ with $g . c . d$. $\left\{n_{1}, n_{2}, \cdots, n_{m}\right\}=1$. We must show that $f \in C^{\infty}$. In [B] J. Boman showed that $f: \boldsymbol{R}^{n} \rightarrow \boldsymbol{R}$ is smooth if and only if $f \circ g$ is smooth for every smooth map $g: \boldsymbol{R}$ $\rightarrow \boldsymbol{R}^{n}$. Hence we may assume that $n=1$, namely $f: \boldsymbol{R} \rightarrow \boldsymbol{R}$. Since g.c.d. $\left\{n_{i}\right\}=1$, there exists a positive integer $p$ such that any integer $r \geqq p$ is written as $r=$ $\sum \alpha_{i} n_{\imath}$ for suitable non-negative integers $\alpha_{\imath}$ and hence $f^{r}=\Pi\left(f^{n_{i}}\right)^{\alpha_{\imath}}$ is smooth.

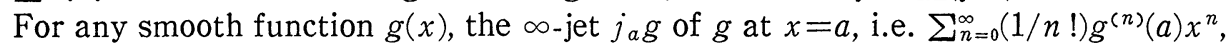
gives an element of the formal power series ring $\boldsymbol{R} \llbracket x \rrbracket$. We say $g$ is flat at $a$ if $j_{a} g=0$. It is easy to show that $f$ is smooth near the non-flat point of $f^{p}$. In fact, assume that $j_{0} f^{p} \neq 0$. Choose an odd prime number $r>p$. Then we have $f^{p}=a x^{n}+\cdots$ and $f^{r}=b x^{m}+\cdots=x^{m} g$ where $g$ is a smooth function with $g(0) \neq 0$. Since $\left(f^{p}\right)^{r}=\left(f^{r}\right)^{p}$ and $m=r l$ for some positive integer $l, f=x^{l} g^{1 / r}$ is smooth near $x=0$. Let $D$ be the set of all non-flat points of $f^{p}$, then $D$ is an open subset of $\boldsymbol{R}$ and $f^{r}$ is flat at every point in $\boldsymbol{R}-D$ for any $r \geqq p$. Now apply Lemma 1 to the ring $\mathscr{R}$ of all continuous functions on $D$ and its subring $\mathcal{S}$ consisting of the restrictions of all continuous functions on $\boldsymbol{R}$ which vanish in $\boldsymbol{R}-D$. Obviously $\mathcal{S}$ has the property $(P)$ in $R$. For any smooth function $g$ on $D,\left\{j_{a} g \mid a \in D\right\}$ can be considered as an element $J(g)$ of $\mathscr{R} \llbracket x \rrbracket$. Now for our $f$ considered as a smooth function on $D, J(f)^{r}=J\left(f^{r}\right) \in \mathcal{S} \llbracket x \rrbracket$ for any $r \geqq p$. So, by Lemma $1, J(f) \in \mathcal{S} \llbracket x \rrbracket$, in other words, for every $n$, the $n$-th derivative $f^{(n)}$ of $f$ is the restriction of a continuous function on $\boldsymbol{R}$ vanishing in $\boldsymbol{R}-D$. The rest of the proof is covered by repeated applications of the following simple lemma.

LEMMA 2. Let $D$ be an open set of $\boldsymbol{R}$. If $f$ and $g$ are both continuous functıons vanishing in $\boldsymbol{R}-D, f$ is differentiable in $D$, and $f^{\prime}(x)=g(x)$ for every $x \in D$, then $f$ is differentiable in the whole $\boldsymbol{R}$ and $f^{\prime}=g$. 
Proof. For $a \in \boldsymbol{R}-D$ and $\varepsilon>0$, there exists $\delta$ such that $|a-x|<\delta$ implies $|g(x)|<\varepsilon$. For any $b$ with $|a-b|<\delta$, let $c$ be the point closest to $b$ among points of $\boldsymbol{R}-D$ between $a$ and $b$. Then since any point $x$ between $b$ and $c$ belongs to $D$ and $\left|f^{\prime}(x)\right|=|g(x)|<\varepsilon$, we have $|f(a)-f(b)|=|f(c)-f(b)| \leqq \varepsilon|c-b|$ $\leqq \varepsilon|a-b|$. So $f$ is differentiable at $a$ and $f^{\prime}(a)=0$. This completes the proof.

\section{Condition for $\left\{\phi_{i}\right\}$.}

Here we give an answer to Joris' Problem. In the sequel, $d(g)$ denotes, for $g=\sum_{n=0}^{\infty} a_{n} x^{n} \in \boldsymbol{R} \llbracket x \rrbracket$, the smallest $n$ such that $a_{n} \neq 0, d(A)=g . c . d .\{d(g) \mid g \in A\}$ for any subset $A \subset \boldsymbol{R} \llbracket x \rrbracket$, and $\left[j_{0} \phi_{1}, j_{0} \phi_{2}, \cdots, j_{0} \phi_{m}\right]$ the subalgebra of $\boldsymbol{R} \llbracket x \rrbracket$ generated by $j_{0} \phi_{i}, i=1,2, \cdots, m$.

THEOREM 3. Let $\phi_{i}: \boldsymbol{R} \rightarrow \boldsymbol{R}$ be smooth functions with $\phi_{i}(0)=0$ for $i=1,2, \cdots, m$. Then the following (1) and (2) are equivalent.

(1) For any continuous function $f: \boldsymbol{R}^{n} \rightarrow \boldsymbol{R}$ with $f(0)=0, f$ is smooth near $x=0$ if and only if $\phi_{i} \circ f$ is smooth near $x=0$ for $i=1,2, \cdots, m$.

(2) $d\left(\left[j_{0} \phi_{1}, j_{0} \phi_{2}, \cdots, j_{0} \phi_{m}\right]\right)=1$.

Proof. As in section 2 we may assume that $n=1$. We consider two more conditions for $\left\{\phi_{i}\right\}$.

(3) There exist smooth functions $F_{\jmath}, j=1,2, \cdots, l$ such that $F_{j}\left(\phi_{1}(x), \phi_{2}(x), \cdots\right.$, $\left.\phi_{m}(x)\right)=x^{n}$, "higher order terms" near $x=0$ with g.c.d. $\left\{n_{1}, n_{2}, \cdots, n_{l}\right\}=1$.

(4) There exist smooth functions $F_{1}$ and $F_{2}$ such that $F_{j}\left(\phi_{1}(x), \phi_{2}(x), \cdots, \phi_{m}(x)\right)$ $=x^{n}$ near $x=0$ with g.c.d. $\left\{n_{1}, n_{2}\right\}=1$.

Proof of $(2) \Leftrightarrow(3)$. This follows from E. Borel's theorem which states that for any element $\tilde{g} \in \boldsymbol{R} \llbracket x \rrbracket$ there exists a smooth function $g(x)$ with $j_{0} g=\tilde{g}$.

Proof of $(3) \Rightarrow(4)$. This is given in Theorem $2[\mathrm{D}]$. Here we give another proof. As in section 2 there exists a positive integer $p$ such that any integer $r \geqq p$ is written as $r=\Sigma \alpha_{j} n$, for some non-negative integers $\alpha$, and hence $\Pi F_{\jmath}\left(\phi_{1}(x), \cdots\right)^{\alpha}{ }_{\jmath}=x^{r}+\cdots$. Then, for any odd integer $n \geqq p$, there exists $\widetilde{F}\left(x_{1}, \cdots, x_{m}\right) \in \boldsymbol{R} \llbracket x_{1}, \cdots, x_{m} \rrbracket$ such that $\tilde{F}\left(j_{0} \phi_{1}, \cdots\right)=x^{n}$ in $\boldsymbol{R} \llbracket x \rrbracket . \quad$ By E. Borel's theorem there exist smooth functions $F$ and $g_{1}$ such that $j_{0} g_{1}=0$ and $F\left(\phi_{1}(x), \cdots\right)$ $\Rightarrow x^{n}+g_{1}(x)$ near $x=0$. We can easily find a smooth function $G$ with $(G \circ F)$ $\left(\phi_{1}(x), \cdots\right)=x^{n}$. In fact, since $y(x)=\left(F\left(\phi_{1}(x), \cdots\right)\right)^{1 / n}$ is smooth and $j_{0} y=x$, we have $x=k(y)=y\left(1+g_{2}(y)\right)$ for suitable smooth functions $k(y)$ and $g_{2}(y)$ with $j_{0} g_{2}=0$. Put $G(Y)=k\left(Y^{1 / n}\right)^{n}$, then $G(Y)=\left(Y^{1 / n}\left(1+g_{2}\left(Y^{1 / n}\right)\right)\right)^{n}=Y\left(1+g_{2}\left(Y^{1 / n}\right)\right)^{n}$ is smooth since $j_{0} g_{2}=0$ and $(G \circ F)\left(\phi_{1}(x), \cdots\right)=G\left(y(x)^{n}\right)=k(y(x))^{n}=x^{n}$ which completes the proof.

Proof of $(4) \Rightarrow(1)$. This follows from Joris' Theorem.

To prove $(1) \Rightarrow(2)$, we need the following algebraic lemma. $g=\sum a_{n} x^{n} \in \boldsymbol{R} \llbracket x \rrbracket$ 
is said to be normal if $a_{d(g)}=1$ and $a_{k d(g)}=0$ for every $k>1$, and for $g \in \boldsymbol{R} \llbracket x \rrbracket$ with $d(g)>0, \llbracket g \rrbracket$ denotes the closed subalgebra generated by $g$, namely $\left\{\sum_{\imath=1}^{\infty} c_{i} g^{2} \mid c_{i} \in \boldsymbol{R}\right\}$.

LEMMA 3. For any subalgebra $A \subset \boldsymbol{R} \llbracket x \rrbracket$ with $d(A)>0$ and any positvve integer $d$ with $d \mid d(A)$, there exists a unique normal elemen $h \in \boldsymbol{R} \llbracket x \rrbracket$ such that $d(h)=d$ and $\llbracket h \rrbracket \supset A$.

Proof. First we prove the following statement.

(i) If $d(h)=d$ and $\llbracket h \rrbracket \cap A \neq\{0\}$ then $\llbracket h \rrbracket \supset A$.

Since the coordinate transformation $y=h(x)^{1 / d}$ induces the isomorphism $\boldsymbol{R} \llbracket x \rrbracket \cong$ $\boldsymbol{R} \llbracket y \rrbracket$ and $h$ corresponds to $y^{d}$, we may assume that $h=x^{d}$. If there exist elements $g=x^{r d}+\cdots+c x^{r d+a}+\cdots \in A$ with $c \neq 0$ and $a \nmid d$, we can choose $g$ having the smallest $a$ in the above representation. Take any $k=x^{s d}+\cdots \in$ $\llbracket x^{d} \rrbracket \cap A \neq\{0\}$, then $g^{s}-k^{r}=b x^{t d}+\cdots+s c x^{s r d+a}+\cdots \in A$ and $s r d+a-t d<a$ contradicting the choice of $g$. This completes the proof of (i). As a corollary we have

(ii) If $d(h)=d\left(h_{1}\right)>0$ and $\llbracket h \rrbracket \cap \llbracket h_{1} \rrbracket \neq\{0\}$ then $\llbracket h \rrbracket=\llbracket h_{1} \rrbracket$.

Now to show the existence of $h$ in the lemma, choose any $g=x^{r d}+\cdots \in A$ and put $h_{1}=g^{1 / r}=x^{d}+\cdots$, then $\llbracket h_{1} \rrbracket \cap A \ni g$ and hence $\llbracket h_{1} \rrbracket \supset A$ by (i). We can easily find real numbers $c_{\imath}$ with $c_{1}=1$ such that $h=\sum_{\imath=1}^{\infty} c_{i} h_{1}^{2}$ is normal. We have also $\llbracket h \rrbracket=\llbracket h_{1} \rrbracket \supset A$ by (ii). To prove the uniqueness of $h$, let $h_{2}$ be any element satisfying the conditions of the lemma, then $\llbracket h \rrbracket=\llbracket h_{2} \rrbracket$ by (ii). If $h-h_{2}$ $\neq 0$ then $d \chi d\left(h-h_{2}\right)$ contradicting the fact $h-h_{2} \in \llbracket h \rrbracket$. This completes the proof of Lemma 3.

Proof of $(1) \Rightarrow(2)$. Suppose $d=d\left(\left[j_{0} \phi_{1}, \cdots\right]\right)>1$. By Lemma 3 there exist $\tilde{h}=x^{d}+\cdots$ and $\widetilde{F}_{i} \in \boldsymbol{R} \llbracket x \rrbracket$ such that $j_{0} \boldsymbol{\phi}_{i}=\widetilde{F}_{i}(\tilde{h})$ for $i=1,2, \cdots, m$. By E. Borel's theorem we have $\phi_{i}(x)=F_{i}(h(x))+g_{i}(x)$ for suitable smooth functions $F_{\imath}, h, g_{\imath}$ with $j_{0} g_{\imath}=0$. So it is sufficient to show that there exists a non-smooth function $f$ for which $h \circ f$ and $g_{i} \circ f$ are smooth. Since $h(x)=x^{d}(1+\cdots)=(x+\cdots)^{d}$, we can find a smooth function $k$ with $h(k(x))=x^{d}$. Now we put $f(x)=k\left(x^{1 / d}\right)$ if $d$ is odd and $f(x)=k(|x|)$ if $d$ is even. Then $f$ is not smooth and $g_{i} \circ f$ is smooth since $j_{0} g_{2}=0$. Moreover $h \circ f$ is smooth, for $h(f(x))=x$ if $d$ is odd and $h(f(x))=x^{d}$ if $d$ is even. This contradicts (1). So the proof of Theorem 3 is completed.

\section{Appendix.}

In this section we discuss about the algorithm of computing $d\left(\left[j_{0} \phi_{1}, \cdots\right]\right)$ by use of jets $j_{0} \phi_{i}$. Let $g$ be an element of $\boldsymbol{R} \llbracket x \rrbracket$ with $d(g)>0$ and $d$ a positive integer with $d \mid d(g)$. Then, applying Lemma 3 to the subalgebra generated by $g$, we can find a unique normal element $h=h(g, d) \in \boldsymbol{R} \llbracket x \rrbracket$ such that $d(h)=d$ and $g=F(h)$ for some $F \in \boldsymbol{R} \llbracket x \rrbracket$. Note that each coefficient of $h(g, d)$ is given 
by some polynomial of $a_{n} / a_{d(g)}$ where $g=\sum a_{n} x^{n}$. Now, put $A=\left[j_{0} \phi_{1}, \cdots\right]$, $d_{A}=d(A)$ and $j_{0} \phi_{i}=\sum_{\jmath=1}^{l_{\imath}} a_{\imath} x^{n_{\imath \jmath}}$ where $a_{\imath j} \neq 0, l_{\imath} \leqq \infty$ and $n_{i 1}<n_{i 2}<\cdots$ for $i=$ $1,2, \cdots, m$. It is clear that g.c.d. $\left\{n_{\imath j} \mid \imath, j\right\} \mid d_{A}$ and $d_{A} \mid$ g.c.d. $\left\{n_{i 1} \mid i\right\}$. In the finite number of integers $d$ such that $d \mid g . c . d .\left\{n_{i 1} \mid i\right\}, d_{A}$ is characterized by the following proposition.

Proposition 1. For any positive integer $d$ with $d \mid$ g.c.d. $\left\{n_{i 1} \mid i\right\}$, we have $d \mid d_{A}$ if and only if $h\left(j_{0} \phi_{1}, d\right)=\cdots=h\left(j_{0} \phi_{m}, d\right)$.

Proof. Assume that $d_{A}=r d$ for some $r$. By Lemma 3, $\llbracket h_{A} \rrbracket \supset A$ for some $h_{A}$ with $d\left(h_{A}\right)=r d$ and hence $j_{0} \phi_{i}=F_{i}\left(h_{A}\right)$ for some $F_{\imath}$. Let $h=h\left(h_{A}, d\right)$, then $h_{A}=F(h)$ for some $F$ and hence $j_{0} \phi_{i}=\left(F_{i} \circ F\right)(h)$. So by the uniqueness of $h\left(j_{0} \phi_{i}, d\right)$, we have $h\left(j_{0} \phi_{i}, d\right)=h$ as desired. Conversely assume that $h\left(j_{0} \phi_{1}, d\right)$ $=\cdots=h$. Then $\llbracket h \rrbracket \ni j_{0} \phi_{i}$ and hence $\llbracket h \rrbracket \supset A$ which implies $d \mid d_{A}$.

The following corollary corresponds to Theorem $3[\mathrm{D}]$.

COROLlary. If $j_{0} \phi_{1}=x^{n_{11}}$ then $d_{A}=$ g.c.d. $\left\{n_{\imath}, i, j\right\}$.

Proof. We have $h\left(j_{0} \phi_{1}, d_{A}\right)=\cdots=h$ and $\jmath_{0} \phi_{i}=F_{i}(h)$ for suitable $F_{i} \in \boldsymbol{R} \llbracket x \rrbracket$. It follows from $j_{0} \phi_{1}=x^{n_{11}}=F_{1}(h)$ that $h=x^{d_{A}}$ and from $\jmath_{0} \phi_{i}=F_{i}\left(x^{d} A\right), d_{A} \mid n_{\imath \jmath}$ which implies $d_{A}=$ g.c.d. $\left\{n_{\imath j} \mid i, j\right\}$.

Proposition 1 gives an algorithm of computing $d_{A}$ by use of jets $j_{0} \phi_{i}$, but it needs an infinite number of procedures to check whether $h\left(j_{0} \phi_{1}, d\right)=\cdots=$ $h\left(j_{0} \phi_{m}, d\right)$ or not even though each $\phi_{\imath}$ is a polynomial. The following Proposition 2 gives a finite algorithm of computing $d_{A}$ for polynomials $\phi_{i}$.

Lemma 4. (i) Let $\mathscr{M}$ be the maximal ideal of $\boldsymbol{C} \llbracket x \rrbracket$ generated by $x$ and $\Phi: \mathscr{M} \rightarrow \boldsymbol{C} \llbracket x \rrbracket^{m}$ a map given by $\Phi(g)=\left(j_{0} \phi_{1} \circ g, \cdots\right)$, then $\Phi$ is $d_{A}$ to 1 map, namely for any $g \neq 0$ there exist exactly $d_{A}$ elements $k$ such that $\Phi(k)=\Phi(g)$.

(ii) Suppose that every $\phi_{i}(x)$ is analytic near $x=0$. Let $\Phi: U \rightarrow C^{m}$ be a map given by $\Phi(x)=\left(\phi_{1}(x), \cdots\right)$ for some neighbourhood $U$ of 0 in $\boldsymbol{C}$, then $\Phi$ is $d_{A}$ to 1 map near $x=0$.

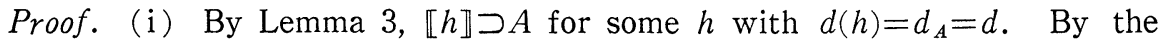
coordinate transformation we may assume that $h=x^{d}$ and hence $j_{0} \phi_{i}=\sum_{j} a_{\imath \jmath} x^{\jmath d}$ for suitable $a_{\imath \jmath}$. By the definition of $d_{A}$ there exists a positive integer $p$ such that for any integer $r \geqq p$ we can find $f \in A$ with $d(f)=r d$ and hence $F \in$ $\boldsymbol{R} \llbracket x_{1}, \cdots, x_{m} \rrbracket$ with $F\left(j_{0} \phi_{1}, \cdots, \jmath_{0} \phi_{m}\right)=x^{r d}$. Therefore $\Phi(k)=\Phi(g)$ if and only if $k^{d}=g^{d}$, that is, $k=\varepsilon_{d}^{n} g$ for $\varepsilon_{d}=\exp \left(2 \pi_{\imath} / d\right)$ and $n=1,2, \cdots, d$.

(ii) Suppose $\Phi(x)=\Phi(y)$. Then $\phi_{\imath}(x)=a_{\imath} x^{r_{i} d}(1+\cdots)=a_{\imath} y^{r_{i} d}(1+\cdots)$ and $y(1+\cdots)=\varepsilon_{r_{i} d}^{n} x(1+\cdots)$ and hence $y=\varepsilon_{r_{i} d}^{n} x+\cdots=y_{\imath n}(x)$. Since $y_{\imath n}(x)$ is holomorphic near $x=0$, it follows from (i) that the number of small solutions $y$ of $\Phi(y)=\Phi(x)$ is $d_{A}$ for small $x \neq 0$.

COROLLARY. The followng condition for $\left\{\phi_{\imath}\right\}$ is equivalent to (1) in Theorem 1 . 
(5) The map $\Phi(g)=\left(j_{0} \phi_{1} \circ g, \cdots\right): \mathscr{M} \rightarrow C \llbracket x \rrbracket^{m}$ is injective.

Proposition 2. Suppose that each $\phi_{i}$ is a polynomial. Let $\phi(x, y)$ be the greatest common divisor of $\left\{\phi_{i}(x)-\phi_{i}(y) \mid i=1,2, \cdots, m\right\}$ in $\boldsymbol{R}[x, y]$, then $d\left(\left[j_{0} \phi_{1}\right.\right.$, $\left.\left.j_{0} \phi_{2}, \cdots, j_{0} \phi_{m}\right]\right)=d(\phi(x, 0))$.

Proof. $\phi(x, y)$ is obtained by using Euclidean algorithm with respect to polynomials of $x$ whose coefficients are rational functions of $y$. Then $\phi(x, a)$ is the greatest common divisor of $\left\{\phi_{i}(x)-\phi_{i}(a) \mid i\right\}$ in $\boldsymbol{R}[x]$ for any real number $a$ with finite exceptions. Since the coefficient of the highest order term of $\phi(x, a)$ is independent of $a$, the solutions $x$ of $\phi(x, a)=0$ depend continuously on a. So $d(\phi(x, 0))$ is equal to the number of solutions $x$ of $\phi(x, a)=0$ such that $x \rightarrow 0$ as $a \rightarrow 0$. This number is equal to the number of common solutions $x$ of $\phi_{i}(x)-\phi_{i}(a)=0, \quad \imath=1,2, \cdots, m$, such that $x \rightarrow 0$ as $a \rightarrow 0$. By Lemma 4 (ii) we have $d(\phi(x, 0))=d_{A}$.

\section{REFERENCES}

[B] Jan Boman, Differentiability of a function and of its compositions with functions of one variable, Math. Scand. 20 (1967), 249-268.

[D] John Duncan, Steven, G. Krantz and Harold R. Parks, Nonlinear conditions for differentiability of functions, J. Analyse Math. 45 (1985), 46-68.

[ J ] HENRi JORIs, Une $C^{\infty}$-application non-immersive qui possède la propriété universelle des immersions, Arch. Math. 39 (1982), 269-277.

Saitama Medical School

KaWAKado, Moroyama

SAITAMA $350-04$, JAPAN

Department of Mathematics

ToKyo Institute of TeCHNOLOGY

Oh-okayama, Meguro-ku

TOKYO 152, JAPAN 